\title{
Correction to: ZNF143 is a regulator of chromatin loop
}

\author{
Zi Wen • Zhi-Tao Huang • Ran Zhang • Cheng Peng
}

Published online: 14 December 2018

(C) Springer Nature B.V. 2018

\section{Correction to: Cell Biology and Toxicology, 2018 https://doi.org/10.1007/s10565-018-9443-z}

There are some errors in Fig. 1. First, owing to a production error, the sub-figure labels $\mathrm{a}, \mathrm{b}$, and $\mathrm{c}$ were absent in the online version, and the proportion number $55.1 \%$ together with its bracket for cell type HelaS3 were messy in the Fig. 1a. Second, owing to the author error, we inadvertently put the GM12878 heatmap on the position of HelaS3 in Fig. 1a, rather than the right HelaS3 heatmap. However, the proportion numbers for HelaS3 heatmap were correct.

We update Fig. 1 below. The figure correction does not affect the text and conclusion in this paper.

The online version of the original article can be found at https://doi.org/10.1007/s10565-018-9443-z

\section{Z. Wen $\cdot$ Z.-T. Huang $\cdot$ C. Peng $(\bowtie)$}

Hubei Key Laboratory of Agricultural Bioinformatics, College of Informatics, Huazhong Agricultural University, Wuhan 430070,

China

e-mail: pengcheng@mail.hzau.edu.cn

\section{R. Zhang}

College of Life Sciences, Hebei Normal University,

Shijiazhuang 050024, China
Publisher's Note Springer Nature remains neutral with regard to jurisdictional claims in published maps and institutional affiliations. 

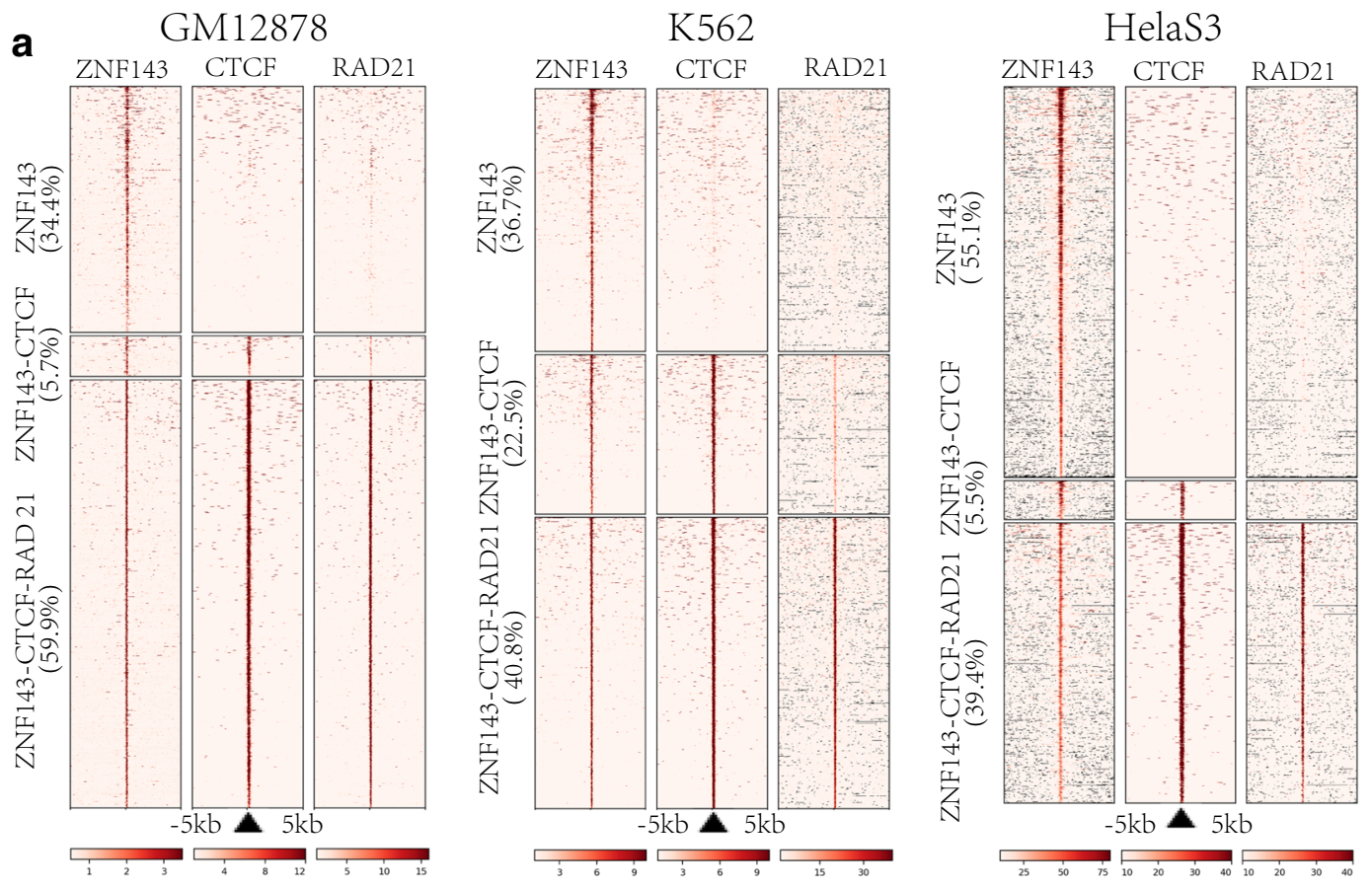

b CTCF(motif)
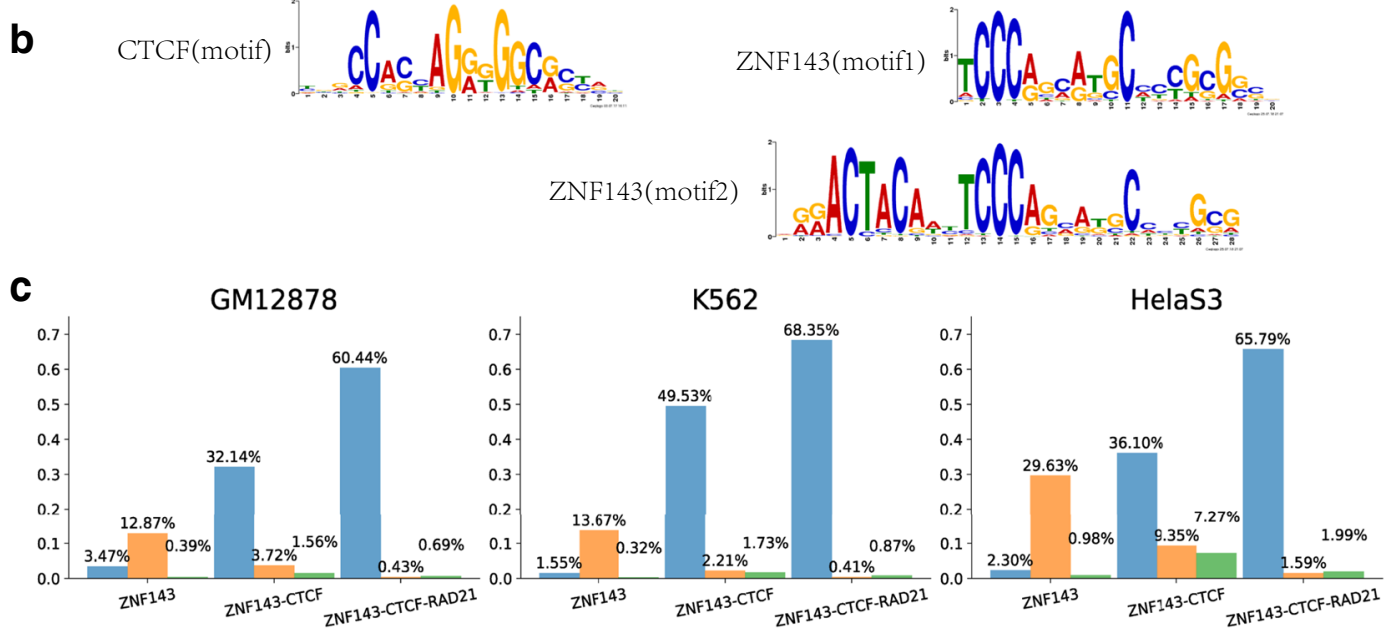

$$
\text { CTCF(motif) ZNF143(motif1) CTCF-ZNF143(motif1) }
$$

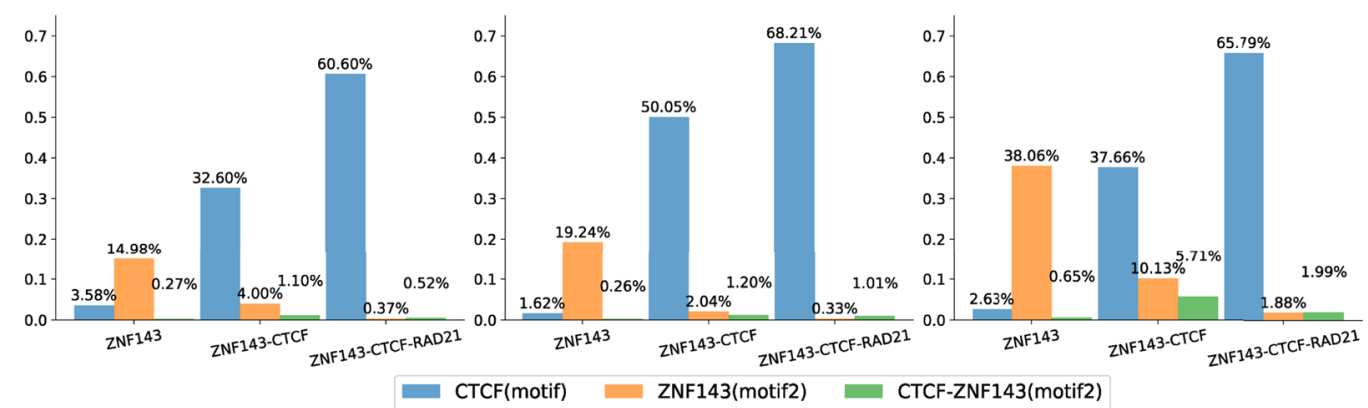

Fig. 1 